\title{
Investigation of Clostridioides (Clostridium) difficile in a community hospital in Southern Ontario, Canada
}

\author{
Devon S. Metcalf; ${ }^{1}$ Robert Clayborne; ${ }^{1}$ Wendy James; ${ }^{1}$ Gabriella Mallia; ${ }^{2}$ \\ Joyce Rousseau; ${ }^{2}$ Melissa Skinner; ${ }^{1}$ John TarBush; ${ }^{1}$ J. Scott Weese ${ }^{2}$ \\ ${ }^{1}$ Guelph General Hospital, Guelph, Ontario \\ ${ }^{2}$ Department of Pathobiology, University of Guelph, Guelph, Ontario \\ at the time the work was conducted. \\ Corresponding author: \\ J. Scott Weese, Dept. of Pathobiology, Ontario Veterinary College, University of Guelph, Guelph, Ontario N1G2W1 \\ jsweese@uoguelph.ca
}

\begin{abstract}
Background: In response to Clostridioides (Clostridium) difficile infections (CDI), infection prevention and control practices in hospital settings tend to focus on symptomatic patients, potentially neglecting other sources of $C$. difficile. The purpose of the study was to identify epidemiological connections between $C$. difficile positive patients to explore the possibility of transmission occurring. This would allow an assessment of IPAC practices to ensure resources were being optimized and targeted to the most appropriate strategies to prevent transmission.

Methods: C. difficile was isolated and characterized from 125 patient stool specimens. Isolates were subjected to toxin profiling and ribotyping. Patient locations in the hospital were mapped and epidemiological connections between patients with the same $C$. difficile ribotype were assessed.

Results: A total of 47 distinct ribotypes were identified, with the most common being ribotype 027/NAP1. Of the 41 cases identified as hospital-associated, only four (9.8\%) of the cases could be epidemiologically linked to another patient with known CDI.

Conclusions: A small minority of hospital-associated infections were found to have an epidemiological link to another known case of CDI suggesting transmission from known cases is rare. This suggested that current IPAC practices were effective in preventing transmission from symptomatic patients, but other sources of $C$. difficile are potentially unrecognized.
\end{abstract}

\section{KEYWORDS}

C. difficile; Ribotyping; Transmission; Hospital

\section{INTRODUCTION}

Clostridioides (Clostridium) difficile is the leading cause of antibiotic-associated diarrhea and a common cause of healthcare-associated infections [1]. Infection prevention and control (IPAC) practices, such as the use of personal protective equipment and the use of sporicides for environmental disinfection, tend to target symptomatic patients with $C$. difficile infection $(\mathrm{CDI})$ because they have been identified as important sources of $C$. difficile and pose a potential risk for transmission to other patients $[2,3]$. However, transmission to hospitalized patients may occur from a variety of sources in addition to symptomatic patients with CDI. Asymptomatically colonized patients, environmental sources such as contaminated surfaces or equipment, or the healthcare workers providing care may also be a source for transmission, or alternatively, the patients may be colonized prior to admission $[4,5,6]$. Despite transmission potentially occurring from a variety of sources and enhanced IPAC practices often only targeting symptomatic patients and the rooms they occupy, other potential sources are often left unaddressed.
Asymptomatically colonized patients are typically not recognized since testing formed stool for $C$. difficile is not recommended [7] and the contribution of asymptomatically colonized patients to the transmission of $C$. difficile remains unclear. Colonization rates in hospitalized patients have been reported to be low, between four to $13 \%[8,9,10]$, and isolating colonized patients has led to a reduction in hospital-associated cases of CDI [11]. Colonized patients are themselves at a greater risk of developing CDI than noncolonized patients [5].

Environmental sources such as shared patient equipment, computers in clinical care areas, medication carts and even laundered linens have been shown to be contaminated with spores and may also play a role in transmission $[12,13]$. C. difficile spores have also been found in areas not involved in direct patient care, such as physician and nurse work areas [12]. Aithinne et al. (2018) [14] demonstrated that C. difficile can even persist in toilet bowl water after multiple flushes and can be aerosolized during flushing, potentially allowing spores 
to settle and persist on surfaces patients may have contact with. As such, many possible sources of exposure exist in hospital settings.

In recent years, investigations into $C$. difficile in hospital environments have suggested that, under non-outbreak conditions, transmission onward from symptomatic patients isn't as common as previously thought. Eyre et al. (2013b) [15] found only 35\% of C. difficile cases were genetically related to a previous case, despite apparent epidemiological links. Walker et al. (2012) [16] also found that the majority of cases (66\%) could not be linked to other known cases. These suggest that there might be overdiagnosis of hospital-associated transmission and outbreaks in situations where unrelated strains are causing disease in an epidemiologically similar fashion. Identifying the sources of $C$. difficile and how transmission occurs is complicated and remains poorly understood.

The objectives of this study were to isolate and characterize $C$. difficile from patients diagnosed with CDI at a healthcare facility over a two-year study period and to identify any epidemiological connections between patients that could support the possibility of transmission occurring. This would allow an assessment of IPAC practices to ensure resources are being optimized and targeted to the most appropriate strategies to prevent transmission.

\section{METHODS}

\section{Specimen collection}

The study took place in a 182-bed community hospital located in Southern Ontario, Canada. The hospital provides care for inpatients in medicine, surgery, obstetrics, paediatrics, stepdown and intensive care units. C. difficile positive specimens from all inpatient and emergency department patients were included for study. All specimens came from patients who met the case definition for CDI [7]. All patients exhibited diarrhea defined as three or more watery or loose stools in a 24-hour period and tested positive for $C$. difficile by a polymerase chain reaction (PCR) based diagnostic test following the manufacturer's instructions (GenXpert ${ }^{\circledR}$ C. difficile/Epi Assay, Cepheid, Sunnyvale, CA) in the hospital microbiology laboratory. All specimens testing positive from June 2014 June 2016 were included. The specimens were stored at $-80^{\circ} \mathrm{C}$ until at least 10 specimens were available for sending to a microbiology research laboratory at the University of Guelph. This study was approved by the research ethics board at the participating hospital and the University of Guelph.

\section{Case classification}

Hospital-associated infections (HAIs) were defined as cases of $\mathrm{CDI}$ in a patient with diarrhea having an onset of symptoms at least 72 hours after admission or within four weeks of a previous hospitalization, when they had not had a C. difficile infection in the previous eight weeks. Infections attributed to another healthcare (other HAIs) facility were defined as cases of $\mathrm{CDI}$ in a patient with the onset of symptoms less than 72 hours into admission and having been exposed to another healthcare facility within the last four weeks. Infections classified as community-acquired (CAIs) or indeterminate were defined as cases of CDI in a patient with the onset of symptoms less than 72 hours after admission and no known exposure to a healthcare facility within the previous four weeks, or where the source of infection could not be determined, respectively. Cases were classified as a relapse if symptoms recurred within eight weeks of the previous infection.

\section{C. difficile culture}

Stools specimens were refrigerated at $4{ }^{\circ} \mathrm{C}$ and cultured within 24 hours of receipt from the hospital. Approximately $200 \mathrm{mg}$ of the stool specimen was immersed in $9 \mathrm{ml}$ of $C$. difficile moxalactam norfloxacin broth with $0.1 \%$ sodium taurocholate and incubated anaerobically at $37^{\circ} \mathrm{C}$ for seven days. Cultures were alcohol shocked (at a 1:1 ratio with anhydrous ethanol) for one hour for spore selection, centrifuged and plated onto CDMN plates (Oxoid, Nepean, Canada). The plates were incubated anaerobically at $37^{\circ} \mathrm{C}$ for 48 hours $\mathrm{C}$. difficile was initially identified by colony morphology, characteristic odour, and a positive I-proline aminopeptidase activity test (Pro Disc, Key Scientific Products, Stamford, TX, USA).

\section{DNA extraction}

DNA was extracted using a commercial kit following the manufacturer's instructions (Instagene Matrix, Biorad, Richmond, CA). Briefly, a $10 \mu$ l loopful of the culture was suspended in $1 \mathrm{ml}$ sterile water in a $1.5 \mathrm{~mL}$ microcentrifuge tube, centrifuged at $12000 \times \mathrm{g}$ for 60 seconds and the supernatant was discarded. The pellets were re-suspended in $200 \mu \mathrm{l}$ of Instagene Matrix, vortexed briefly and incubated at $56^{\circ} \mathrm{C}$ for 30 minutes. The tubes were then incubated at $100^{\circ} \mathrm{C}$ for eight minutes, centrifuged at $12000 \times \mathrm{g}$ for two minutes and the supernatants were removed to a fresh tube and stored at $-20^{\circ} \mathrm{C}$ for future use.

\section{Detection of $C$. difficile toxins}

PCR to detect the toxin A gene (tcdA), the toxin B gene $(t c d B)$, and the binary toxin genes $(c d t A$ and $c d t B)$ was performed as previously described [17]. Amplification reactions were performed using a Mastercycler ${ }^{\circledR}$ pro $S$ Thermal Cycler (Eppendorf, Mississauga, Ontario) and the following cycling parameters: 10 minutes at $94^{\circ} \mathrm{C}, 35$ cycles of 50 seconds at $94^{\circ} \mathrm{C}, 40$ seconds at $54^{\circ} \mathrm{C}, 50$ seconds at $72^{\circ} \mathrm{C}$, and a final extension of three minutes at $72^{\circ} \mathrm{C}$. The $25 \mu \mathrm{l}$ reaction contained $2 \mu \mathrm{l}$ DNA, $1.0 \mu \mathrm{l}$ of $25 \mathrm{mM} \mathrm{MgCl}$,

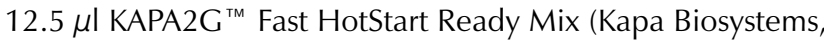
Boston, MA), and primers as previously described [17]. PCR products were resolved on a $1.5 \%$ agarose gel and visualized using GelRed DNA stain (Biotium, Hayward, CA).

\section{Capillary ribotyping}

Briefly, $200 \mathrm{ng}$ of purified DNA is used with $0.2 \mu \mathrm{M}$ of the $16 \mathrm{~S}$ (5'-GTGCGGCTGGATCACCTCCT-3') and $23 \mathrm{~S}$ (5'-CCCTGCACCCTTAATAACTTGACC-3') primers. 
The 16 S primer was labelled at the $5^{\prime}$ end with a fluorescent label. Amplification reactions were performed using a Mastercycler ${ }^{\circledR}$ pro S Thermal Cycler (Eppendorf, Missisauga, Ontario) with the following cycling parameters: 15 minutes at $95^{\circ} \mathrm{C}, 24$ cycles of 60 seconds at $95^{\circ} \mathrm{C}, 60$ seconds at $57^{\circ} \mathrm{C}, 60$ seconds at $72^{\circ} \mathrm{C}$, and a final extension of 30 minutes at $72^{\circ} \mathrm{C}$. The $25 \mu \mathrm{l}$ reaction contained $2 \mu \mathrm{l}$ DNA,

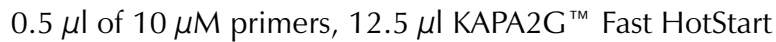
Ready Mix (Kapa Biosystems, Boston, MA).

PCR products were analyzed using the Webribo server (https://webribo.ages.at). Ribotypes identified as international ribotypes based on comparison to reference strains were assigned the appropriate numerical designation and an internal laboratory number was assigned for all other isolates.

\section{Analysis}

The patients who tested positive for $C$. difficile during their admission were mapped in the hospital by physical location (unit) from the day of admission until discharge. The patients were mapped to three medicine units, two surgical units, an intensive care unit, a step-down unit, a family birthing unit and the emergency department. An epidemiological link that would support the possibility that transmission had occurred was defined as one patient who goes on to develop CDI after spending a minimum of 12 hours on the same unit as another patient who had tested positive for $C$. difficile within the previous eight weeks, with both patients infected with the same ribotype. Basic patient demographic data, reason for hospital visit/admission and recent antibiotic usage were recorded.

\section{RESULTS}

During the 25-month study period, 125 specimens were isolated from 119 patients diagnosed with CDI and C. difficile was isolated from all samples. The classification of infections and summary of toxin profiles are summarized in Table 1.

All 125 isolates were successfully ribotyped and classified into 47 distinct ribotypes, with two subtypes of ribotype 002 being identified (002 and 002/2) and three subtypes of ribotype 014 being identified (014/0, 014/4 and 014/5). Twenty-two isolates belonged to 19 newly identified ribotypes. The most common ribotypes are summarized in Table 2. The 41 HAls belonged to 18 distinct ribotypes.

Eight of the 14 cases classified as a relapse had the initial infection diagnosed at another facility therefore the specimens from the initial cases were unobtainable and the ribotype is unknown. Of the remaining six relapse cases, only 4/6 (67\%) had the same ribotype identified as the initial infection. Two cases had an initial isolate classified as a different ribotype compared to the relapse episode (Table 3).

\section{TABLE 1: Summary of the classification and toxin profiles of $C$. difficile isolates.}

\begin{tabular}{|c|c|c|c|}
\multirow{3}{*}{ Classification } & & Total & Percent (\%) \\
\cline { 2 - 4 } & HAI & $41 / 125$ & 32.8 \\
\cline { 2 - 4 } & Other HAI & $17 / 125$ & 13.6 \\
\cline { 2 - 4 } & CAI & $53 / 125$ & $14 / 125$ \\
\cline { 2 - 4 } & Relapse & 11.2 & 125 \\
\hline
\end{tabular}

\begin{tabular}{|c|c|c|c|c|c|}
\hline \multirow[t]{4}{*}{ Toxin Profile } & $\begin{array}{c}\text { Toxin A } \\
\text { (tcdA) }\end{array}$ & $\begin{array}{c}\text { Toxin B } \\
(\operatorname{tcdB})\end{array}$ & $\begin{array}{l}\text { Binary toxin } \\
\text { (cdtA/cdtB) }\end{array}$ & & \\
\hline & + & + & - & $85 / 125$ & 68.0 \\
\hline & - & + & - & $5 / 125$ & 4.0 \\
\hline & - & - & - & $1 / 125$ & 0.8 \\
\hline
\end{tabular}

\begin{tabular}{|c|c|c|c|c|c|}
\hline Ribotype & $\begin{array}{c}\text { Total No. } \\
\text { of isolates (\%) }\end{array}$ & Total No. of HAls & $\begin{array}{c}\text { Total No. of } \\
\text { CA-CDIs }\end{array}$ & $\begin{array}{l}\text { Total No. of } \\
\text { other-HAIs }\end{array}$ & $\begin{array}{l}\text { Total No. } \\
\text { of Relapses }\end{array}$ \\
\hline 020 & $7(5.6)$ & 5 & 2 & 0 & 0 \\
\hline $014 / 0$ & $7(5.6)$ & 0 & 3 & 2 & 2 \\
\hline $002 / 2$ & $5(4.0)$ & 3 & 0 & 1 & 1 \\
\hline 012 & $4(3.2)$ & 3 & 1 & 0 & 0 \\
\hline
\end{tabular}


TABLE 3: Relapse cases that differ in ribotype

between the initial and relapse episode of CDI.

\begin{tabular}{|c|c|c|}
\hline $\begin{array}{c}\text { Initial Case } \\
\text { Ribotype }\end{array}$ & $\begin{array}{c}\text { Relapse Case } \\
\text { Ribotype }\end{array}$ & $\begin{array}{c}\text { Time between } \\
\text { positive test }\end{array}$ \\
\hline 075 & $014 / 0$ & 55 days \\
\hline 027 (NAP1) & GGH13 ${ }^{1}$ & 48 days \\
\hline${ }^{1}$ Internal laboratory designation \\
\hline
\end{tabular}

Demographic and clinical characteristics, including the indication for previous antibiotic use and the admitting diagnosis, are summarized in Table 4.

For the 41 cases identified as hospital-associated, an epidemiological link to another patient known to have, or have had a recent CDI, was investigated. Only 4/41 (9.8\%) of the HAls could be epidemiologically linked to another patient with CDI when ribotype was considered (Table 5). The remaining $37 / 41$ (90\%) cases either shared no time on the same unit as another patient with $\mathrm{CDI}$, or was found to have a different ribotype than a patient on the same unit at the same time.

\section{DISCUSSION}

In this study, only 9.8\% (4/41) of infections classified as hospitalassociated had an epidemiological link to another known case of $\mathrm{CDI}$ suggesting transmission from known cases is rare. On the onset of diarrhea, a series of infection prevention precautions were initiated. In accordance with best practice [7], contact precautions were initiated, which included the accommodation of patients in private rooms with dedicated toileting facilities and all staff and visitors were required to don gloves and a gown prior to any contact with the patient or the patient environment. A sporicidal disinfectant was used for twice-daily environmental cleaning of the patient room and bathroom until the patient was symptom-free for a specified period of time or discharged. In this study, very few cases developed as a result of onward transmission from known cases and therefore the additional precautions initiated for suspect and confirmed cases of CDI were presumably effective in preventing transmission.

Antimicrobial stewardship may be more effective, compared to additional precautions, in reducing CDIs as this strategy would address patients already colonized, or those becoming colonized by an unidentified source as opposed to strictly relying on targeting prevention strategies to recognized exogenous sources.

Recent antibiotic exposure is a well-known risk factor for CDI [18]. Not surprisingly, the majority of patients (70\%) had recognized recent exposure to antibiotics prior to the onset

\begin{tabular}{|c|c|c|}
\hline Characteristics & Range & Mean (years of age) \\
\hline \multirow[t]{2}{*}{ Age (years of age) } & $15-98$ & 66 \\
\hline & Total & Percentage (\%) \\
\hline Female & $75 / 125$ & 60.0 \\
\hline
\end{tabular}

\begin{tabular}{|r|c|c|}
\hline \multicolumn{2}{|l|}{ Admitted from } \\
\hline Home & $100 / 125$ & 80.0 \\
\hline Long term care home & $22 / 125$ & 17.6 \\
\hline Another hospital & $2 / 125$ & 1.6 \\
\hline Unknown & $1 / 125$ & 0.8 \\
\hline
\end{tabular}

\begin{tabular}{|r|c|c|}
\hline Recent Antibiotic use $^{1}$ & $90 / 125$ & 72.0 \\
\hline Cephalosporin & $53 / 90$ & 58.9 \\
\hline Fluoroquinolone & $32 / 90$ & 35.6 \\
\hline Penicillin & $26 / 90$ & 28.9 \\
\hline$>1$ antibiotic & $55 / 90$ & 61.1 \\
\hline No antibiotics & $30 / 125$ & 24.0 \\
\hline Proton pump inhibitors & $75 / 125$ & 60.0 \\
\hline
\end{tabular}

\begin{tabular}{|r|c|c|}
\hline Antibiotic Indication $^{2}$ & & \\
\hline Pneumonia & $23 / 125$ & 18.4 \\
\hline Urinary tract infection & $18 / 125$ & 14.4 \\
\hline Sepsis & $10 / 125$ & 8.0 \\
\hline Surgical prophylaxis & $10 / 125$ & 8.0 \\
\hline Cellulitis & $7 / 125$ & 5.6 \\
\hline
\end{tabular}

\begin{tabular}{|r|c|c|}
\hline Admitting diagnosis & & \\
\hline Diarrhea & $58 / 125$ & 46.4 \\
\hline Cardiac concerns $^{3}$ & $7 / 125$ & 5.6 \\
\hline Pneumonia & $6 / 125$ & 4.8 \\
\hline Urinary tract infection & $5 / 125$ & 4.0 \\
\hline
\end{tabular}

1 Antibiotic use within the previous four weeks prior to CDI diagnosis

2 Indication for antibiotic prescribed within four weeks prior to $\mathrm{CDI}$ diagnosis

3 Cardiac concerns including congestive heart failure, rapid atrial fibrillation, myocardial infarction

\section{TABLE 5: Summary of the CDI cases that had an epidemiological link to another known CDI case.}

\begin{tabular}{|c|c|c|l|}
\hline Case No. & Date & Ribotype & Epidemiological Link \\
\hline 39 & Feb. 2015 & 027 & Patient spent seven days on the same unit as another case of ribotype 027 \\
\hline 40 & Feb. 2015 & $002 / 2$ & Patient spent 16 days on the same unit as another case of ribotype 002/2 \\
\hline 70 & Aug. 2015 & 020 & Patient spent nine days on the same unit as another case of ribotype 020 \\
\hline 78 & Sep. 2015 & 020 & Patient spent nine days on the same unit as another case of ribotype 020 \\
\hline
\end{tabular}


of symptoms of CDI. The two most frequent indications for antibiotic therapy were pneumonia and urinary tract infections (UTIs). CDI following pneumonia and UTIs are common and have been shown to have a higher in-hospital mortality rate and to result in a longer length of stay [19]. Antimicrobial stewardship practices targeting pneumonia and UTI cases may reduce overall $\mathrm{CDI}$ rates $[20,21]$.

The sources of $C$. difficile in the majority of the infections classified as hospital-associated in this study are unknown. They may include other asymptomatically colonized patients or staff, an environmental source, or the patients who develop infections may be colonized prior to admission. Patients may also have had contact with other parts of the hospital not identified in this study, highlighting a potential limitation. The possibility of acquisition of $C$. difficile from an unrecognized location outside of the units the patients were admitted to (e.g., operating rooms, diagnostic imaging rooms) cannot be ruled out. C. difficile positive patients may have contaminated those locations creating an unrecognized opportunity for transmission.

The role of asymptomatically colonized patients in transmission remains unclear. Eyre et al (2013a) [4] demonstrated that onward transmission from asymptomatically colonized patients to cases of CDI was likely very rare and Kong et al. 2019 [22] confirmed this, finding only $6 \%$ of new CDls could be linked solely to colonized patients. Curry et al (2013) [23] reported a higher rate finding 29\% of new CDI cases in their study could be linked to asymptomatically colonized patients. Differences in molecular techniques used and baseline $\mathrm{CDI}$ rates in the respective patient populations may account for these differences. Overall, neither infected patients nor asymptomatically colonized patients may be the most common source of incident CDIs in hospitals.

The most common ribotype identified in this study was ribotype 027 (NAP 1) (Table 2) which has previously been reported to be a common type identified in healthcare settings, although it is becoming less frequent $[24,25]$. This study found a broader variety of ribotypes than previously reported in similar studies. The 125 isolates were classified into 47 distinct ribotypes while Aptekorz et al. (2017) [24] classified 108 isolates from 15 different hospitals into only eight ribotypes and found $7 / 108(6.5 \%)$ to be non-typable. Furuya-Kanamori et al. (2016) [26] found greater variety, classifying 324 isolates into over 90 ribotypes, but also included isolates from colonized patients. These studies were conducted in different countries in varying patient populations and used different ribotyping techniques compared to this study, which may contribute to the varying results. In Canada, in a non-outbreak setting, 46 isolates from a hospital were classified into 10 different ribotypes by Labbé et al. (2008) [27] and Martin et al., (2008) [28] classified over 1,000 isolates provided by 21 diagnostic laboratories into only 39 distinct ribotypes. The reason why such a heterogeneous population of C. difficile was identified in this study isn't clear, but may indicate that the exposures to $C$. difficile may be from a broad range of sources in this patient population.

Another limitation of this study is that only one molecular typing technique (ribotyping) was performed to differentiate strains. All of the cases with epidemiological links to other cases were among the top five most common ribotypes identified in this study. A more discriminatory typing technique, such as whole genome sequencing, may have further differentiated these strains. If anything, typing using higher resolution methods would reduce the number of likely HAls even further, if isolates of the same ribotype were identified as distinct.

The identification of patients colonized on admission or throughout the duration of stay was not investigated during this study, but is an opportunity for future research. In the future, as suggested by O'Hagan and McDonald (2018) [29], sampling of healthcare workers' hands and the collection of environmental samples could provide estimates of transmissions, which may be prevented by a focus on hand hygiene or improved environmental cleaning practices.

Two cases identified as relapses had different ribotypes isolated from the specimen compared to the initial isolates. The patients may have been infected with more than one ribotype of C. difficile highlighting another limitation of this study. Only one colony was characterized per specimen and additional strains may have been present and were not identified. Alternatively, the patients may have become infected with a new strain of C. difficile within the timeframe to classify the case as a relapse. Given that none of these patients had an epidemiological link to another patient with $\mathrm{CDI}$ with the same strain during the time period between the initial and relapse infections, if infection with a new strain had occurred, the source is unknown. Further investigation into the sources of $C$. difficile in cases classified as relapses is warranted.

Without the ribotyping data, 75\% (31/41) of the hospitalassociated cases of CDI would have an epidemiological link based only on time and location within the hospital. This could result in efforts to improve or enhance the IPAC practices focusing on those symptomatic patients potentially wasting IPAC resources better directed at other potential sources of transmission.

This study identified a heterogeneous population of C. difficile in this patient population with ribotype 027 (NAP1) identified as the most common ribotype. The majority of new CDIs in these patients could not be epidemiologically linked to other patients with active CDIs. Other sources of $C$. difficile should be investigated and identified to ensure that the IPAC practices being implemented are the most strategic and effective.

\section{REFERENCES}

1. Leffler, D., Lamont, T. (2015). Clostridium difficile Infection. New England Journal of Medicine, 372, 1539-1548. doi: 10.1056/ NEJMra1403772

2. Dubberke, E., Reske, K., Olsen, M., McMullen, K., Mayfield, J., McDonald, L., Fraser, V. (2007). Evaluation of Clostridium difficileassociated disease pressure as a risk factor for $C$. difficile-associated disease. Archives of Internal Medicine, 167(10), 1092-1097. doi: 10.1001/archinte.167.10.1092

3. Bobulsky, G., Al-Nassir, W., Riggs, M., Sethi, A., Donskey, C. (2008). Clostridium difficile skin contamination in patients with C. difficile-associated disease. Clinical Infectious Diseases, 46(3), 
447-450. doi: 10.1086/525267

4. Eyre, D., Griffiths, D., Vaughan, A., Golbchik,T ., Acharya, M., O`Connor, L., Crook, D., Walker, A., Peto, T. A. (2013). Aysmptomatic Clostridium difficile Colonization and Onward Transmission. PLoS One, 8(11), e78445. doi: 10.1371/journal. pone.0078445

5. Zacharioudakis I., Zervou, F., Pliakos, E., Mylonakis, E. (2015). Colonization with toxigenic $C$. difficile upon hospital admission, and risk of infection: a systematic review and meta-analysis. American Journal of Gastroenterology, 110(3), 381-390. doi: 10.1038/ajg.2015.22

6. MacDougall, L., Broukhanski, G., Simor, A., et al. (2018). Comparison of qPCR versus culture for the detection and quantification of Clostridium difficile environmental contamination. PLoS One, 13(8), e0201569. doi: 10.1371/journal.pone.0201569

7. Ontario Agency for Health Protection and Promotion, Provincial Infectious Diseases Advisory Committee (PIDAC). (2013) Annex C - Testing, Surveillance and Management of Clostridium difficile. Annexed to: Routine Practices and Additional Precautions in All Health Care Settings. Retrieved from https://www.publichealthontario.ca/-/media/documents/cdiff-testing-surveillance-management.pdf?la $=$ en

8. Barbut, F., Petit, J. C. (2001). Epidemiology of Clostridium difficileassociated infections. Clinical Microbiology and Infection, 7, 405410. PMID: 11591202

9. Loo, V., Bourgault, A. M., Poirier, L., Lamothe, F., Michaud, S. et al. (2011). Host and pathogen factors for Clostridium difficile infection and colonization. New England Journal Medicine, 365, 1693-1703. doi: 10.1056/NEJMoa1012413

10. Guerrero, D., Becker, J., Eckstein, E., Kundrapu, S., Deshpande, A., Sethi, A., Donskey, C. (2013). Asymptomatic carriage of toxigenic Clostridium difficile by hospitalized patients. Journal of Hospital Infections, 85(2), 155-158. doi: 10.1016/j.jhin.2013.07.002

11. Longtin Y., Paquet-Bolduc, B., Gilca R. et al., (2016). Effect of Detecting and Isolating Clostridium difficile carriers at hospital admission on the incidence of $C$. difficile infections: A quasiexperimental controlled study. JAMA Internal Medecine, 176, 796-804. doi: 10.1001/jamainternmed.2016.0177

12. Dumford, D., Nerandzic, M., Eckstein, B., Donskey, C. (2009). What is on that keyboard? Detecting hidden environmental reservoirs of Clostridium difficile during an outbreak associated with North American pulsed-field gel electrophoresis type 1 strains. American Journal of Infection Control, 37(1), 15-19. doi: 10.1016/j.ajic.2008.07.009

13. Tarrant J, Jenkins, R., Laird, K. (2018). From ward to washer: The survival of Clostridium difficile spores on hospital bed sheets through a commercial UK NHS healthcare laundry process. Infection Control Hospital Epidemiology, 39(12), 1406-1411. doi: 10.1017/ice.2018.255

14. Aithinne, K., Cooper, C., Lunch, R., Johnson, D. (2018). Toilet plume aerosol generation rate and environmental contamination following bowl water inoculation with Clostridium difficile spores. American Journal of Infection Control, 47(5), 515-520. doi: 10.1016/j.ajic.2018.11.009

15. Eyre, D., Cule, M., Wilson, D., et al. (2013b). Diverse sources of $C$. difficile infection identified on whole-genome sequencing. New England Journal of Medicine, 369(13), 1195-1205. doi: 10.1056/NEJMoa1216064

16. Walker, A., Eyre, D., Wyllie, D., Dingle, K., Harding, R., O'Connor, L., Griffiths, D. et al. (2012). Characterization of Clostridium difficile Hospital Ward-Based Transmission Using Extensive Epidemiological Data and Molecular Typing. PLoS Medicine, 9(2), e1001172. doi: 10.1371/journal.pmed.1001172

17. Persson, S., Torpdahl, M., Olsen, K. (2008). New multiplex PCR method for the detection of Clostridium difficile toxin $\mathrm{A}(\mathrm{tcd} A)$ and toxin $B(t c d B)$ and the binary toxin $(c d t A / c d t B)$ genes applied to a Danish strain collection. Clinical Microbiology and Infection, 14, 1057-1064. doi: 10.1111/j.1469-0691.2008.02092.x

18. Mora Pinzon, M., Buie, R., Liou, J., et al. (2018). Outcomes of Community and Healthcare-onset Clostridium difficile infections. Clinical Infectious Diseases, 68(8), 1343-1350. doi: 10.1093/cid/ ciy715

19. Becerra, M., Becerra, B., Banta, J., Safdar, N. (2015). Impact of Clostridium difficile infection among pneumonia and urinary tract infection hospitalizations: an analysis of the Nationwide Inpatient Sample. BMC Infectious Diseases, 15, 254:263. doi: 10.1186/ s12879-015-0925-9

20. Wenisch, J., Equiluz-Bruch, S., Fudel, M., et al. (2014). Decreasing Clostridium difficile Infections by an Antimicrobial Stewardship Program That Reduces Moxifloxacin Use. Antimicrobial Agents and Chemotherapy, 58(9), 5019-5083. doi: 10.1128/ AAC.03006-14

21. Ge, I., H. Fevrier, C. Conell, et al. (2018). Reducing risk of Clostridium difficile infection and overall use of antibiotic in the outpatient treatment of urinary tract infection. Therapeutic Advances in Urology, 10, 283-293. doi: 10.1177/1756287218783871

22. Kong, L., Eyre, D., Corbeil, J., et al. (2019). Clostridium difficile: Investigating Transmission Patterns Between Infected and Colonized Patients Using Whole Genome Sequencing. Clinical Infectious Disease, 68(2), 204-209. doi: 10.1093/cid/ciy457

23. Curry, S., Muto, C., Schlackman, J. et al. (2013). Use of multilocus variable number of tandem repeats analysis genotyping to determine the role of asymptomatic carriers in Clostridium difficile transmission. Clinical Infectious Diseases, 57, 1094-1102. doi: $10.1093 / \mathrm{cid} / \mathrm{cit} 475$

24. Aptekorz, M., Szczegielniak, A., Wiechula, B., Harmanus, C., Kuijper, E., Martirosian, G. (2017). Occurrence of Clostridium difficile ribotype 027 in hospitals of Silesia, Poland. Anaerobe, 45, 106-113. doi:10.1016/j.anaerobe.2017.02.002

25. Katz, K., Golding, G., Baekyung, et al. (2018). The evolving epidemiology of Clostridium difficile infection in Canadian hospital during a postepidemic period (2009-2015). Canadian Medical Association Journal, 190(25), E758-765. doi: 10.1503/ cmaj.180013

26. Furuya-Kanamori, L., Riley, T., Paterson, D., et al. (2016). Comparison of Clostridium difficile ribotypes circulating in Australian Hospital and Communites. Journal of Clinical Microbiology, 55, 216-225. doi:10.1128/JCM.01779-16

27. Labbé, A.C., Poirier, L., MacCannell, D., et al. (2008). Clostridium difficile Infections in Canadian Tertiary Care Hospital before and during a Regional Epidemic Associated with the BI/NAP1/027 Strain. Antimicrobial Agents and Chemotherapy. 52(9), 31803187. doi: 10.1128/AAC.00146-08

28. Martin, H., Willey, B., Low, D., et al. (2008). Characterization of Clostridium difficile Strains Isolated from Patients in Ontario, Canada, from 2004 to 2006. Journal of Clinical Microbiology, 46(9), 2999-3004. doi: 10.1128/JCM.02437-39. O'Hagan, J., McDonald, L. C. (2018). The Challenges of Tracking Clostridium difficile to its source in hospitalized patients. Clinical Infectious Diseases, 68(2), 210-212. doi: 10.1093/cid/ciy461 * 